\title{
Editorial Message: Special Issue on Advances in Evolutionary Fuzzy Systems
}

\author{
Chin-Wang Tao ${ }^{1} \cdot$ Chen-Chia Chuang $^{1} \cdot$ Hsu-Chih Huang ${ }^{1}$
}

Published online: 15 November 2016

(C) Taiwan Fuzzy Systems Association and Springer-Verlag Berlin Heidelberg 2016

As the guest editors of the special issue on advances in evolutionary fuzzy systems, we would like to publish the latest, innovative, and outstanding research results in the International Journal of Fuzzy Systems (IJFS) with a very short review period. This issue was called for papers and participation in the special IJFS section of the 2015 international conference on fuzzy theory and its applications (iFUZZY 2015) held in Yilan, Taiwan, over November 18-20, 2015. A total of 19 papers were submitted to the special IJFS session. They were initially reviewed and asked to provide the oral presentations by the selected reviewers. Based on the three review criteria on technical contributions, novelty, and completeness, nine papers were provisionally accepted and requested for quality improvements according to the reviewers' comments and suggestions. Seven papers have finally been accepted for publication in the IJFS special issue on advances in evolutionary fuzzy systems after substantial revisions.

All the seven papers are collected together in the current issue in order to illustrate the main technical achievements of the special IJFS session in iFUZZY 2015. These articles provide novel, interesting, and useful results covering "A Generalization of Rand and Jaccard Indices with Its Fuzzy Extension", "Contextual Improvement Planning by FuzzyRough Machine Learning: A Novel Bipolar Approach for Business Analytics", "A Novel Fuzzy Logic Model for Pseudo-Relevance Feedback-Based Query Expansion", "Composite Learning Fuzzy Control of Uncertain Nonlinear Systems", "Real-Time Self-Localization of a Mobile Robot by Vision and Motion System", "Design of a DSPBased PD-like Fuzzy Controller for Buck DC-DC Converters", and "Experimental Validation for Fuzzy Control of Servo Pneumatic Artificial Muscle Driven by Metal Hydride". This editorial message not only delineates the paper submission, rigorous review, and quality assurance of the special IJFS session papers, but also significantly appreciates the authors' patience for paper revisions according to the comments.
Chin-Wang Tao

cwtao@niu.edu.tw

1 Department of Electrical Engineering, National Ilan University, No. 1, Sec. 1, Shennong Rd, Yilan City, Yilan County, Taiwan, ROC 